\title{
COMPLEX FUNCTION ALGEBRAS
}

\author{
BY \\ H. S. BEAR
}

1. Introduction. This paper is a study of closed subalgebras of the algebra $C(X)$ of all continuous complex-valued functions on a compact Hausdorff space $X$, where $C(X)$ is topologized by uniform convergence on $X$ and the algebraic operations are carried out pointwise.

By the maximal ideal space of an algebra we mean the space of continuous complex homomorphisms of the algebra with the weak* topology. (For our purposes it is usually more convenient to consider the homomorphisms than the maximal ideals which are their kernels.) For a subalgebra $A$ of $C(X)$ the evaluation mapping $\left(x \rightarrow e_{x}\right.$, where $\left.e_{x}(f)=f(x)\right)$ is a homeomorphism of $X$ onto a subset of the maximal ideal space of $A$. We shall accordingly always regard the underlying space $X$ to be a subset of the maximal ideal space in such a way that the identity function on $X$ is the evaluation mapping into the maximal ideal space. Particular emphasis is given to the case in which the algebra is defined on the whole of its maximal ideal space. This is a natural emphasis for several reasons. First, the maximal ideal space is always available (theoretically, at least); second, an algebra defined on its maximal ideal space has several pleasant properties not always available (e.g., a nowhere zero function in the algebra has an inverse in the algebra); and third, the study of such algebras provides a logical continuation of the classification process started by Gelfand's Theorem [2] which shows that a class of Banach algebras are (isomorphic and isometric to) algebras of functions on their spaces of maximal ideals.

In $\$ 2$ we show that for any subalgebra $A$ of $C(X)$ there is a unique minimal closed subset $E$ of $X$ such that any continuous function which agrees on $E$ with a function of $A$ is in $A$. Theorems are proved which show that the study of any algebra can effectively be reduced to the case where this minimal set $E$ is all of $X$. In $\$ 3$ maximal subalgebras are studied, and in $\S 4$ several weaker maximality conditions are investigated.

The following notation and conventions are used throughout:

" $A$ is an algebra on $X$ " means " $X$ is a compact Hausdorff space, $A$ is a proper closed subalgebra of $C(X)$, the constant functions belong to $A$, and for any two points of $X$, there is a function in $A$ which takes different values at the two points."

The maximal ideal space of $A$ is denoted $\Sigma(A)$, and $Z(f)=\{x: f(x)=0\}$ for each $f$ in $A$. The sets $Z(f)$, for $f \in A$ and $f \not \equiv 0$, are called the zero-sets of $A$. The support of $f$ is the closure of the complement of $Z(f)$.

Received by the editors May 21, 1957. 
The author would like to acknowledge his indebtedness to Professors Bishop, Helson, Royden, and Yood, and in particular to Professor J. L. Kelley who supervised the research.

2. The essential set of an algebra. The fundamental lemma of this section is Corollary 1 to Theorem 1 below. Theorem 1 is due to Bertram Yood, and replaces the author's direct proof of the corollary.

Theorem 1. If $B$ is a commutative topological algebra, and $A$ is a proper closed subalgebra, then there is a unique closed ideal I of $B$ such that

(a) $I \subset A$,

(b) $I$ is not contained in any larger ideal of $B$ which is contained in $A$.

Proof. Let $g$ be the set of all closed ideals of $B$ which are subsets of $A$. The ideal generated by $U g$ is an ideal of $B$ contained in $A$, hence its closure $I$ belongs to $\mathscr{I}$ since $A$ is closed. It is clear that $I$ is not contained in any larger ideal of $B$ which is contained in $A$, and that $I$ is the only ideal of $B$ satisfying (a) and (b).

Corollary 1. If $A$ is a closed subalgebra of $C(X)$, then there is a unique minimal closed subset $E$ of $X$ such that any continuous function zero on $E$ is in $A$. An equivalent statement is the following: there is a unique minimal closed set $E$ such that for any continuous $f$ and any $g \in A$, if $f=g$ on $E$, then $f \in A$.

Proof. Let $I$ be the largest closed ideal of $C(X)$ contained in $A$. Then $I=\{f: f=0$ on $E\}$, for some closed set $E$, and $E$ is uniquely minimal since $I$ is uniquely maximal.

Definition. If $A$ is an algebra on $X$, the minimal closed subset $E$ of $X$ whose existence is guaranteed by Corollary 1 will be called the essential set of $A$ in $X$, or if no confusion can arise, simply the essential set of $A$. If $E=X$, then we shall say that $A$ is an essential algebra on $X$.

Corollary 2. If $A_{1} \subset A_{2} \subset C(X)$, and $E_{1}, E_{2}$ are the essential sets for $A_{1}, A_{2}$ respectively, then $E_{2} \subset E_{1}$.

Proof. Let $I_{1}, I_{2}$ be the largest closed ideals of $C(X)$ contained in $A_{1}, A_{2}$ respectively; then $E_{1}, E_{2}$ are the hulls of $I_{1}, I_{2}$. By the construction of the $I$ 's used in the proof of Theorem 1 , it is clear that $I_{1} \subset I_{2}$, and hence $E_{2} \subset E_{1}$.

For an algebra $A$ on $X$ and a closed subset $E$ of $X$, we will denote by $A \mid E$ the algebra on $E$ of restrictions to $E$ of functions in $A$. $(A \mid E)^{-}$will denote the completion of $A \mid E$ in the sense of uniform convergence on $E$.

Theorem 2. If $E$ is the essential set for $A$, then $(A \mid E)^{-}=A \mid E \neq C(E)$.

Proof. We must show that if $f_{n} \in A$ and $f_{n}|E \rightarrow f| E$, then $f \in A$. Any function $g_{n}$ which equals $f_{n}$ on $E$ is necessarily in $A$, and there exists a sequence $\left\{g_{n}\right\}$ of such functions which converges uniformly to $f$ on $X$; hence $f \in A$. If $A \mid E=C(E)$, then $A=C(X)$, which is contrary to our standing assumptions on $A$. 
We will next study the relationship between the maximal ideal spaces of algebras and their essential sets. First we prove the following theorem, due to $\mathrm{H}$. Royden, which is extremely useful in deciding what the maximal ideal space of a given algebra is.

THEOREM 3 (Royden). If $A$ is a separating algebra of continuous functions on the compact space $X$, then $X=\Sigma(A)$ if and only if for each finite set $\left\{f_{1}, \cdots, f_{n}\right\}$ of functions in $A$ which have no common zeros, there are functions $e_{1}, \cdots, e_{n}$ in $A$ such that $e_{1} f_{1}+\cdots+e_{n} f_{n}=1$.

Proof. Note that for $n=1$, the above says that the algebra is inverseclosed, which is clearly a necessary condition. Suppose the above condition holds, and $M$ is a maximal ideal of $A$. If $\cap\{Z(f): f \in M\}=0$, then there are a finite number of functions $f_{1}, \cdots, f_{n}$ in $M$ such that $\cap\left\{Z\left(f_{i}\right): i=1, \cdots, n\right\}$ $=0$, since $X$ is compact. Then the fact that there are functions $e_{1}, \cdots, e_{n}$ in $A$ such that $\sum e_{i} f_{i}=1$ contradicts the assumption that $M$ is a proper ideal. Hence all $f$ in $M$ must vanish at some $x_{0}$, and since $M$ is maximal, $M=\left\{f: f\left(x_{0}\right)=0\right.$ and $\left.f \in A\right\}$. That is, each maximal ideal is represented as a point of $X$, and different ideals correspond to different points since $A$ is separating.

Now suppose that $X=\Sigma(A)$, and $f_{1}, \cdots, f_{n}$ have no common zeros. Let $M$ be the ideal generated by $f_{1}, \cdots, f_{n}$; i.e.: $M=\left\{g_{1} f_{1}+\cdots+g_{n} f_{n}: g_{i} \in A\right\}$. If $M$ is a proper ideal, then $M$ is contained in a maximal ideal

$$
M_{0}=\left\{f: f\left(x_{0}\right)=0\right\} .
$$

Let $g_{1}, \cdots, g_{n}$ be functions in $A$ such that $g_{1}\left(x_{0}\right) \neq 0$, and $g_{2}\left(x_{0}\right)=\cdots$ $=g_{n}\left(x_{0}\right)=0$. Since $\sum g_{i}\left(x_{0}\right) f_{i}\left(x_{0}\right)=0, f_{1}\left(x_{0}\right)=0$. The same procedure can be repeated to show that $f_{i}\left(x_{0}\right)=0$ for $i=1,2, \cdots, n$, which contradicts the assumption that the $f_{i}$ have no common zeros. Therefore, $M$ is not a proper ideal, and there are functions $g_{1}, \cdots, g_{n} \in A$ such that $g_{1} f_{1}+\cdots+g_{n} f_{n}=1$.

We have shown that in considering any subalgebra $A$ of $C(X)$, we may restrict $A$ to that subset of $X$, the essential set, which carries the structure of the algebra. We next show that this process of restriction leaves us an algebra defined on its natural domain, the maximal ideal space, if the original algebra was defined on its maximal ideal space. While it is not true in general that $(A \mid F)^{-}$has $F$ as maximal ideal space for an arbitrary closed subset $F$ of $X$, we show in Theorem 5 that the maximal ideal space is preserved by this sort of restriction if $F$ is a zero set of $A$.

THEOREM 4. If $A$ is an algebra of functions on $X$, and $E$ is the essential set for $A$, then $\Sigma(A \mid E)=E$ if and only if $\Sigma(A)=X$.

Proof. We have already noticed that $A \mid E$ is a closed subalgebra of $C(E)$. First assume $\Sigma(A)=X$. Any continuous homomorphism $\phi$ on $A \mid E$ extends to a continuous homomorphism on $A$, and hence is representable as an evalu- 
ation: for each $f \in A, \phi(f \mid E)=f\left(x_{0}\right)$ for some $x_{0} \in X$. If $x_{0} \notin E$, then there is a function $g \in A$ which is zero on $E$ and 1 at $x_{0}$, and hence $0=\phi(0)=\phi(g \mid E)$ $=g\left(x_{0}\right)=1$, a contradiction. Therefore, each homomorphism on $A \mid E$ is an evaluation at a point of $E$.

Now assume that $\Sigma(A \mid E)=E$, and that $A$ contains all extensions to $X$ of any function in $A \mid E$. We use Royden's theorem to show $\Sigma(A)=X$. If $f_{1}, \cdots, f_{n}$ have no common zeros then they have no common zeros on $E$, and there are functions $e_{1}, \cdots, e_{n}$ in $A$ such that $e_{1} f_{1}+\cdots+e_{n} f_{n}=1$ on $E$. Let $g$ be the complex conjugate of $\sum e_{i} f_{i} ; g \in A$, since $g=1$ on $E$, and $\sum g e_{i} f_{i}$ is real and non-negative on $X$, and bounded away from zero on a neighborhood $U$ of $E$. Let $k_{1}, \cdots, k_{n}$ be functions which are zero on $E$ (hence functions in $A$ ) and such that $\sum k_{i} f_{i}=1$ off $U$. This is possible since $A \mid X \sim U$ $=C(X \sim U)$ and the maximal ideal space of the restricted algebra is $X \sim U$. Let $h$ be the conjugate of $\sum k_{i} f_{i}$; then $\sum h k_{i} f_{i}$ is zero on $E$, real, and nonnegative. The sum $r=\sum g e_{i} f_{i}+\sum h k_{i} f_{i}=\sum\left(g e_{i}+h k_{i}\right) f_{i}$ is real, positive, and 1 on $E$, and hence $r^{-1} \in A$, and $\sum r^{-1}\left(g e_{i}+h k_{i}\right) f_{i}=1$. The functions $r^{-1}\left(g e_{i}+h k_{i}\right)$ are all in $A$.

Theorem 5. If $A$ is an algebra on $X$ and $\Sigma(A)=X$, and $E=Z(\varphi)$ for some $\varphi \in A$, then $\Sigma(A \mid E)^{-}=E$.

Proof. If $\pi$ is a continuous homomorphism on $(A \mid E)^{-}$, then $\pi$ extends to $A$ and hence for $f \in A, \pi(f)=f\left(x_{0}\right)$ for some $x_{0} \in X$. If $x_{0} \notin E$, then $\pi(\varphi \mid E)$ $=\pi(0)=0=\varphi\left(x_{0}\right) \neq 0$.

Theorem 6. If $A$ is an essential algebra on $X$, and $\Sigma(A)=X$, then $X$ is perfect.

Proof. Any algebra contains the characteristic function of any openclosed set in its maximal ideal space [9]. If $X$ had an isolated point, $x_{0}$, then any function zero on $X \sim\left\{x_{0}\right\}$ would be a constant multiple of the characteristic function of $\left\{x_{0}\right\}$, and hence in the algebra, contradicting the fact that $X$ is the essential set.

Note. The preceding theorem would be false without the assumption that $\Sigma(A)=X$. The algebra $A$ of all continuous functions on the closed unit $\operatorname{disc}\{z:|z| \leqq 1\}$ which agree on the rim and at the origin with some function analytic in $\{z:|z|<1\}$ has the rim plus $\{0\}$ as its essential set. The maximal ideal space of $A$ can be pictured as two copies of the unit disc pasted together at the rims and centers. That is, if we denote by $f_{a}$ that analytic function which agrees with $f$ on the rim and at the center, then a homomorphism of $A$ is of the form $\pi(f)=f(z)$, or of the form $\pi(f)=f_{a}(z)$, for some $z$ in the disc. It is clear that $A$ is isomorphic to an algebra on this space of homomorphisms, and it is easy to verify via Theorem 3 that this space is in fact $\Sigma(A)$. The essential set of $A$ in $\Sigma(A)$ is just one copy of the disc, and $A$ on its essential set is just the analytic functions. While it is evident that by its definition $A$ 
is related to the algebra of functions analytic on the disc, it is interesting to see how the algebra of analytic functions is forced into view by the process of first considering the maximal ideal space, and then restricting to the essential set.

Theorem 7. If $A$ is an essential algebra on $X$ and $\Sigma(A)=X$, then $X$ is not completely disconnected, and no open-closed subset of $X$ is completely disconnected.

Proof. If $X$ is completely disconnected, then each point has arbitrarily small open-closed neighborhoods. Let $f$ be an arbitrary continuous function on $X$. For each $x$, let $F_{x}$ be an open-closed neighborhood of $x$ on which the variation of $f$ is less than $\epsilon$. Let $F_{1}, \cdots, F_{n}$ be a finite number of these which cover $X$, and take the $F_{i}$ to be pairwise disjoint. If $x_{i} \in F_{i}$, and $e_{i}$ is the characteristic function of $F_{i}$, then $f\left(x_{1}\right) e_{1}+\cdots+f\left(x_{n}\right) e_{n}$ is an $\epsilon$-approximation to $f$, and hence $f \in A$. That is, $A$ is not a proper subalgebra of $C(X)$. If $X$ had a completely disconnected open-closed subset, then $A$ would not be essential on $X$.

Note. Rudin has shown [6] that there is a proper subalgebra of $C(X)$ for any $X$ which contains a homeomorphic image of the Cantor set. His subalgebras actually have the Cantor set as their essential set, and the above shows that such a subalgebra must always have maximal ideal space different from $X$. Rudin's example is defined on the Riemann sphere.

If $A$ is an algebra on $X$ there is a unique minimal closed subset of $X$, called the Silov boundary of $A$, on which each function in $A$ assumes its maximum modulus. A point $x_{0}$ belongs to the Šilov boundary if and only if for each neighborhood $U$ of $x_{0}$ there is a function $f$ in $A$ such that max $\{|f(x)|: x \in X\}$ is assumed only at points in $U$. It is natural to assume that the Silov boundary is closely related to the structure of a function algebra, and that consideration of the boundary is a natural place to attack the problem of a structure. (See, e.g. [7].) The foregoing discussion indicates that no general conclusion can be drawn on the basis of boundary considerations if the algebra is not essential. For example, if $A$ is the algebra of all continuous functions on a 3-dimensional sphere which are analytic on a given disc within the sphere, then the Silov boundary is the whole space, and there is nothing about the boundary to distinguish the algebra from $C(X)$. This same phenomenon can occur in a finitely generated algebra. Let $\Gamma$ be a curve (a continuous 1-1 image of a half-open interval) in 3-space which intersects every horizontal plane in at most one point and whose closure is $\Gamma \cup D$, where $D$ $=\{z:|z| \leqq 1\}$. The algebra generated by the functions $t$ (height), and $z=x+i y$ is the algebra of all functions whose restrictions to the open disc are analytic. The maximal ideal space of this algebra is the space $\Gamma \cup D$, since the essential set is $D$ and it is well known that the algebra of functions on $D$ which are analytic in the interior has $D$ as its maximal ideal space, and 
Theorem 4 says that the whole algebra then has $\Gamma \cup D$ as its maximal ideal space. The Šilov boundary of this algebra is the whole space $\Gamma \cup D$.

Theorem 8. If $X$ is any closed set in the complex plane, and $A$ is the algebra of all uniform limits of rational functions with poles off $X$, then $\Sigma(A)=X$.

Proof. (Suggested by H. Royden.) First note that $A$ is inverse closed; i.e., if $R_{n} \rightarrow f$ and $f$ has no zeros, then $R_{n}$ has no zeros for sufficiently large $n$, and $R_{n}^{-1} \rightarrow f^{-1}$. Let $\pi$ be any continuous homomorphism on $A$, and let $\pi(z)=z_{0}$. If $z_{0} \in X$, and $f(z)=z-z_{0}$, then $f^{-1} \in A$, and $\pi\left(f \cdot f^{-1}\right)=1=0 \cdot \pi\left(f^{-1}\right)$, which is impossible. If $R$ is a rational function, then $\pi(R)=R\left(z_{0}\right)$, and hence $\pi(g)$ $=g\left(z_{0}\right)$ for any limit of rational functions, since $\pi$ is necessarily continuous.

Mergelyan gives an example of a closed nowhere dense subset $X$ of the complex plane such that not every continuous function on this set can be approximated by rational functions [4, p. 19]. Thus the algebra of all functions which are rationally approximable is a proper subalgebra of $C(X)$ which, by the preceding theorem, has $X$ as its maximal ideal space. It follows that there is an essential algebra on a closed subset of $X$.

The importance of this example lies in the fact that the other known examples of subalgebras of $C(X)$ for various compact spaces $X$ are built around an algebra of analytic functions. Thus Rudin's and Wermer's examples $[6 ; 11]$ are the algebras of all functions continuous on the Riemann sphere and analytic off some closed nowhere dense set of positive area. The example destroys the hope of finding, for any subalgebra, an analytic manifold embedded in the maximal ideal space such that all the functions in the algebra are analytic on the manifold, without further geometric restrictions on the maximal ideal space.

\section{Maximal algebras.}

Definition. $A$ is maximal on $X$ if and only if $A$ is a proper subalgebra of $C(X)$ and there is no other proper closed subalgebra of $C(X)$ containing $A$.

We consider below some properties of maximal algebras, and related facts concerning the Silov boundary of an algebra. Recall that if $A$ is an algebra on $X$ and $B$ is the Silov boundary, then $A \mid B$ is a closed subalgebra of $C(B)$ which is isomorphic and isometric to $A$.

Theorem 9. If $A$ is an algebra on $X$ and $B$ is the Šlov boundary of $A$, then $A \mid B \neq C(B)$.

Proof. Assume $A \mid B=C(B)$ and $x_{0} \in X \sim B .(B \neq X$ since $A$ is a proper subalgebra of $C(X)$.) Define the homomorphism $\pi$ on $C(B)$ by $\pi(f)=\tilde{f}\left(x_{0}\right)$, where $\tilde{f}$ is the unique function in $A$ which agrees with $f$ on $B$. Since $\pi$ is continuous, and $\Sigma(C(B))=B, \pi$ can be represented as evaluation at a point of $B: \pi(f)$ $=f\left(x_{1}\right)$ for all $f \in A$. That is, $f\left(x_{0}\right)=f\left(x_{1}\right)$ for all $f \in A$, which contradicts our standing assumption that $A$ separates points of $X$. 
TheOREM 10. If $A$ is an algebra on $X$, and $B$ is the Silov boundary, then any $f \in A$ which is real on $B$ is real on $X$, and $f[B]=f[X]$.

Proof. Suppose there is a point $z_{0} \in f[X] \sim f[B]$. Since $f[B]$ is a compact subset of the real line there is a polynomial $p(z)$ such that $p\left(z_{0}\right)=1$ and $|p(z)|<1 / 2$ on $f[B]$. The function $p(f(z))$ is in $A$ but does not attain its maximum modulus on $B$, which is a contradiction.

Helson and Quigley have investigated maximal subalgebras of $C(X)$ and shown the existence of an essential set for a maximal algebra, and that the restriction of a maximal algebra to its essential set is maximal [3]. Theorem 12 and Corollary 1 below are due to Helson and Quigley. The proof given here is new and will depend on Theorem 11 and Lemma 1 below, which will also be used later.

Theorem 11. If $A$ is an algebra on $X, F$ is the support of a member $\varphi$ of $A$ and $A \mid F$ is dense in $C(F)$, then $A$ contains all continuous functions zero on $Z(\varphi)$. (That is, $Z(\varphi)$ contains the essential set.)

Proof. Since the theorem is trivially true if $Z(\varphi)$ has no interior, we restrict our attention to the case $Z(\varphi)^{0} \neq 0$. Let $f$ be any continuous function which is zero on a neighborhood of $Z(\varphi)$. We will show that $f \in A$, and since any function zero on $Z(\varphi)$ can be uniformly approximated by functions zero on a neighborhood of $Z(\varphi)$, the theorem will follow. Let $g_{0}=\varphi^{-1} f$ if $\varphi \neq 0$, and $g_{0}=0$ elsewhere; then $g_{0}$ is continuous. Let $g_{n} \in A$ and $g_{n}\left|F \rightarrow g_{0}\right| F$. Then $\varphi g_{n} \in A$, and $\varphi g_{n} \rightarrow \varphi g_{0}=f$, so $f \in A$.

The fact that $A \mid F$ is dense in $C(F)$ does not imply that $X \sim F^{0}$ contains the essential set without the hypothesis that $F$ is the support of a function in $A$. For example, if $A$ is the algebra of functions on the unit circle which have analytic continuations to the unit disc, then $A \mid F$ is dense in $C(F)$ for every proper closed set $F$.

Lemma 1. If $A$ is an essential maximal algebra on $X$, then $A \mid F$ is dense in $C(F)$ for each closed $F \neq X$.

Proof. For any closed $F \neq X$, there is a function $\varphi$ such that $\varphi$ is zero on $F$, and $\varphi \notin A$, since $A$ is essential. Since $A$ and $\varphi$ generate $C(X)$, polynomials $g_{0}+g_{1} \varphi+\cdots+g_{n} \varphi^{n}, g_{i} \in A$, are dense in $C(X)$ and hence their restrictions to $F$ are dense in $C(F)$. The restrictions to $F$ of such polynomials are in $A \mid F$, however, since $\varphi=0$ on $F$.

Corollary. If $A$ is an essential maximal algebra on $X$ then the Silov boundary $B$ of $X$ is identical with $X$.

Proof. $A \mid B$ is closed, so $A \mid B=C(B)$, if $B \neq X$, but this contradicts Theorem 9. 
A more general form of the corollary above was discovered independently by Paul Civin [1].

Theorem 12 (Helson-Quigley). If $A$ is an essential maximal algebra on $X$ and $\varphi$ is a member of $A$ which is zero on an open set, then $\varphi$ is identically zero.

Proof. Assume $\varphi \in A$ and $\varphi$ is zero on an open set but not identically zero. If $F$ is the support of $\varphi$, then $A \mid F$ is dense in $C(F)$ by Lemma 1 , and hence $A$ is not essential by Theorem 11 .

CoROLlary 1. If $A$ is an essential maximal algebra, then the only real functions in $A$ are the constants.

Proof. If $\varphi$ is a real function in $A$, then every continuous function constant on the same sets as $\varphi$ is in $A$. If $\varphi$ is not constant, there is a function in $A$ zero on an open set and not identically zero.

Corollary 2. If $A$ is an essential maximal algebra on $X$ and $F$ is a closed proper subset of $X$ with nonempty interior, then $A \mid F$ is not closed in $C(F)$.

Proof. If $A \mid F$ is closed, $A \mid F=C(F)$, and if $F$ has nonvoid interior, there are functions in $A$ zero on an open subset of $F$ and not identically zero.

Corollary 3. If $A$ is an essential maximal algebra on $X$ and $A$ is inverseclosed (nowhere zero functions in $A$ have inverses in $A$ ), then each nowhere zero function is characterized by its absolute value.

Proof. Specifically, we mean by the above that if $f, g \in A$ and $|f| \equiv|g|>0$, then $f=\alpha g$, where $\alpha$ is a constant. Let $h=f g^{-1}$; then $\bar{h}=f^{-1} g$, and $h, \bar{h}$ are in $A$ and have their ranges contained in the unit circle. Any continuous function on the unit circle can be uniformly approximated by polynomials in $z$ and $\bar{z}$, so if $h$ is not a constant, there would be a function in the algebra which was zero on an open set but not identically zero.

4. Maximality. In this section we investigate several variants of the idea of a maximal algebra. The fact that none of the known maximal algebras are maximal on their maximal ideal spaces leads us to consider a modification of the notion of a maximality which preserves our emphasis on algebras defined on their maximal ideal spaces. We then consider the problem of extending a given algebra to one maximal with respect to some property of the algebra.

Definition. $A$ is an extremal algebra if and only if there is no other proper subalgebra $A_{1}$ of $C(\Sigma(A))$ such that $A \subset A_{1}$ and $\Sigma\left(A_{1}\right)=\Sigma(A)$.

An algebra maximal on its maximal ideal space is certainly extremal, but an algebra maximal on some subset of its maximal ideal space need not be extremal. For example, the algebra $A$ of functions analytic on the open unit disc and continuous on the closed disc is a maximal algebra on the circle, but is not maximal on its maximal ideal space, the disc, or even extremal. The algebra of all continuous extensions to the unit disc of functions analytic on 
$\{z:|z|<1 / 2\}$ and continuous on $\{z:|z| \leqq 1 / 2\}$ contains $A$ and has the unit disc as maximal ideal space. Likewise, there is no $a$ priori reason to assume that an extremal algebra is maximal, either on its maximal ideal space or on a smaller set. In any case we can show that extremal algebras share several significant properties with maximal algebras, and that the reduction to essential algebras proceeds as before.

Lemma 2. If $A$ is extremal, and $E$ is the essential set of $A$ in $\Sigma(A)$, then $A \mid E$ is extremal.

Proof. We have already noted that $\Sigma(A \mid E)=E$. If $A \mid E$ is properly conrained in $A_{1}$, and $\Sigma\left(A_{1}\right)=E$, then the algebra $A_{2}$ of all extensions to $\Sigma(A)$ of functions in $A_{1}$ has maximal ideal space $\Sigma(A)$, and properly contains $A$.

We may henceforth consider only essential extremal algebras, since no generality is lost by this reduction.

Lemma 3. If $A$ is an essential extremal algebra, then $A \mid F$ is dense in $C(F)$ for every zero-set $F$ of $A$ in $\Sigma(A)$.

Proof. We have shown that $\Sigma(A \mid F)^{-}=F$ if $F$ is a zero set of $A$. So if $(A \mid F)^{-} \neq C(F)$, then we can form the algebra of all extensions to $\Sigma(A)$ of $(A \mid F)^{-}$and obtain a proper subalgebra of $C(\Sigma(A))$ containing $A$ and having $\Sigma(A)$ as maximal ideal space.

Theorem 13. If $A$ is an essential extremal algebra, then $A$ has no zero divisors.

Proof. Suppose $\varphi_{1}, \varphi_{2} \in A, \varphi_{1} \not \equiv 0 \not \equiv \varphi_{2}$, and $\varphi_{1} \varphi_{2} \equiv 0$. Let $E_{1}=Z\left(\varphi_{1}\right)$ and $F_{2}$ be the support of $\varphi_{2}$; then $F_{2} \subset E_{1}$. By Lemma $3, A \mid E_{1}$ is dense in $C\left(E_{1}\right)$ and hence dense in $C\left(F_{2}\right)$. Theorem 11 then shows that $A$ is not essential, which is a contradiction.

Corollary. If $A$ is an essential extremal algebra, then there is some point $x_{0}$ of $\Sigma(A)$ such that every function in $A$ which is zero on a neighborhood of $x_{0}$ is identically zero.

Proof. If for each $x \in \Sigma(A)$ there were a function zero on a neighborhood of $x$, then by covering $\Sigma(A)$ with a finite number of these neighborhoods, and considering the corresponding functions, we could construct zero divisors.

Definition. $A$ is a regular algebra if and only if for each two disjoint closed subsets $F_{1}$ and $F_{2}$ of $\Sigma(A)$ there is a function in $A$ which is zero on $F_{1}$ and one on $F_{2}$.

Theorems 12 and 13 point out a relationship between the following important unanswered questions.

(1a) Can any proper closed subalgebra of $C(X)$ be extended to an algebra maximal on $X$ ?

(1b) Can any algebra be extended to an extremal algebra? 
(2) Are there any regular function algebras other than algebras of all continuous functions?

If the answer to either (1a) or (1b) is yes, then the answer to (2) is no, since regular algebras are obviously neither maximal nor extremal.

As indicated above, nothing is known about the general problem of when a given algebra can be extended to a maximal or to an extremal algebra. If each function in the algebra has a property which is definitely not enjoyed by all continuous functions and which persists under uniform limits, then the algebra can be extended to one maximal with respect to this property by a simple Zorn's lemma argument. In the case considered by Rudin [6] the algebra maximal with respect to the critical property (viz., that the range of the real part of each function in the algebra be connected) is in fact a maximal algebra. We carry out below such a maximizing process with respect to the property of having a given Silov boundary which is not the whole space. Maximality with respect to boundary is certainly not sufficient to imply maximality, since the analytic functions on the unit disc can not be extended to a larger algebra which has the rim as Silov boundary [7].

Lemma 4. If $A$ is an algebra on $X$ with boundary $B$, and $B \neq X$, then $A$ is contained in an algebra maximal with respect to having boundary $B$.

Proof. If $\mathfrak{N}$ is a nest of algebras containing $A$ and having boundary $B$, and $A_{0}$ is the uniform closure of $\cup \Re$, then we will show that $A_{0}$ has boundary $B$, and hence $A_{0}$ is a proper subalgebra of $C(X)$, and hence $A$ is contained in a maximal such algebra. Let $f_{n} \in \cup \mathfrak{N}$ and $\left\|f_{n}\right\|=\left|f_{n}\left(x_{n}\right)\right|, x_{n} \in B$, and $x_{n} \rightarrow x_{0} \in B$, and $\left\|f_{n}-f\right\|<1 / n$. Then $\left\|f_{n}\right\|-1 / n<\|f\|<\left\|f_{n}\right\|+1 / n$ and $\left|f_{n}\left(x_{n}\right)\right|$ $-1 / n<\left|f\left(x_{n}\right)\right|<\left|f_{n}\left(x_{n}\right)\right|+1 / n$; hence ||$f||-\left|f\left(x_{n}\right)\right| \mid<2 / n$, for each $n$, and $\|f\|=\left|f\left(x_{0}\right)\right|$. Hence $B$ is the boundary of the closure of $U \mathfrak{N}$.

Lemma 5. If $\mathfrak{R}$ is a nest of algebras with common maximal ideal space $X$, and $A_{0}$ is the closure of $\cup \mathfrak{N}$, then $\Sigma\left(A_{0}\right)=X$.

Proof. Let $\phi$ be a continuous homomorphism on $A_{0} . \phi$ is evaluation at some $x_{0}$ for all $f$ in each $A \in \mathfrak{N}$, and this $x_{0}$ is independent of $A$ since $\mathfrak{N}$ is a nest. If $f \in A_{0}$, and $f_{n} \rightarrow f, f_{n} \in \cup \Re$, then $\phi\left(f_{n}\right)=f_{n}\left(x_{0}\right) \rightarrow \phi(f)=f\left(x_{0}\right)$.

Theorem 14. If $\Sigma(A)=X$ and the boundary of $A$ is $B \neq X$, then $A$ is contained in an algebra $A_{0}$ with maximal ideal space $X$ and boundary $B$ which is maximal with respect to these conditions.

Proof. Lemmas 4 and 5 show that a Zorn's lemma argument works under the above assumptions.

\section{REFERENCES}

1. Paul Civin, A maximum modulus property of maximal subalgebras, Proc. Amer. Math. Soc. vol. 10 (1959) pp. 51-54.

2. I. Gelfand, Normierte Ringe, Rec. Math. (Mat. Sbornik) N.S. vol. 9 (1941) pp. 3-24. 
3. H. Helson and F. Quigley, Maximal algebras of continuous functions, Proc. Amer. Math. Soc. vol. 8 (1957) pp. 111-114.

4. S. N. Mergelyan, Uniform approximations to functions of a complex variable, Amer. Math. Soc. Translations, no. 101.

5. Walter Rudin, Boundary values of continuous analytic functions, Proc. Amer. Math. Soc. vol. 7 (1956) pp. 808-811.

6. - Subalgebras of spaces of continuous functions, Proc. Amer. Math. Soc. vol. 7 (1956) pp. 825-830.

7. - Analyticity and the maximum modulus principle, Duke Math. J. vol. 20 (1953) pp. $449-457$.

8. G. E. Šilov, On rings of functions with uniform convergence, Ukrain. Mat. Ž. vol. 33 (1951) pp. 404-411 (Russian).

9. - On decomposition of a commutative normed ring in a direct sum of ideals, Mat. Sbornik N.S. vol. 32 (74) (1953) pp. 353-364 (Russian).

10. John Wermer, On algebras of continuous functions, Proc. Amer. Math. Soc. vol. 4 (1953) pp. 866-869.

11. - Polynomial approximation on an arc in $C^{3}$, Ann. of Math. vol. 62 (1955) pp. $269-270$.

12. - Subalgebras of the algebra of all complex-valued continuous functions on the circle, Amer. J. Math. vol. 78 (1956) pp. 225-242.

University of CALIFornia, Berkeley, Calif. 